\title{
Diagnosis and Inference of ADHD
}

\author{
I.Jemima Bibiyana*, K.Krishnaveni**, E.Radhamani*** \\ *(M.Phil Scholar, Department of Computer Science, Sri S.R.N.M.College, Sattur, Tamil Nadu \\ ** (Associate Professor, Department of Computer Science, Sri S.R.N.M.College, Sattur, Tamil Nadu \\ *** (Research Scholar, Department of Computer Science, Sri S.R.N.M.College, Sattur, Tamil Nadu \\ Corresponding Author: I.Jemima Bibiyana
}

\begin{abstract}
Attention Deficit Hyperactivity Disorder (ADHD) may occur due to abnormal brain development or Brain injuries occurring before, during or after birth. Any brain damage or brain injury may cause abnormality in the growth of caudate nucleus and other brain parts. The children with ADHD may have caudate volume reduction. So, in this paper, to predict the prevalence of ADHD, three different segmentation techniques namely Fuzzy CMeans Segmentation, Region Based Active Contour Segmentation and Threshold Based Segmentation are proposed to extract the caudate nucleus from the MRI brain images. The segmentation results are compared and analyzed based on various performance metrics and found that the Fuzzy C-Means Segmentation algorithm performs well in terms of region continuity, computation time, and accuracy. Instead of going for medication or any other behavioral treatment the brain imaging techniques may easily identify the occurrence of ADHD and assist the neurologists to learn more about this disorder and hence take preventive measures to overcome this
\end{abstract} problem.

Keywords: ADHD, Caudate Nucleus, Fuzzy C-Means, MRI, Region Based Active Contour and Threshold.

\section{INTRODUCTION}

Attention Deficit Hyperactivity Disorder (ADHD) is a mental disorder which causes various hyperactive and troublemaking behaviors. One of the main causes of ADHD among the children is the uncontrollable growth of cells in the brain. The human brain controls and systemizes the overall functions and activities in the human body which can be divided into three main regions: forebrain, midbrain and hindbrain. The forebrain (pros encephalon) is made up of thalamus, hypothalamus, cerebrum, and pineal gland and other features. The center of the brain which is called midbrain (mesencephalon) is composed of a portion of the brainstem. The hindbrain (rhomb encephalon) consists of the remaining brainstem as well as cerebellum and pons [1]. Brain cells can be broken into two groups namely gray matter and white matter. Gray matter consists of neurons and glial cells which controls the brain activity. The White matter is also made up of neurons that can connect gray matter to each other brain parts and protect the neurons.

\subsection{Brain STRUCTURE}

Human brain contains cerebral cortex, frontal lobes, cortex, limbic system, cerebrum, thalamus, hippo thalamus, hippocampus and reticular activating system. The existing research reveals that the brain size of children with ADHD will be $3 \%$ to $4 \%$ smaller than the brains of children without ADHD. i.e, the ADHD Children will have smaller frontal lobes, temporal gray matter, caudate nucleus, and cerebellum which are the brain regions involved in concentration, impulse control, inhibition, and motor activity. The human brain anatomy with the regions which are affected by ADHD is shown in Fig 1[1].

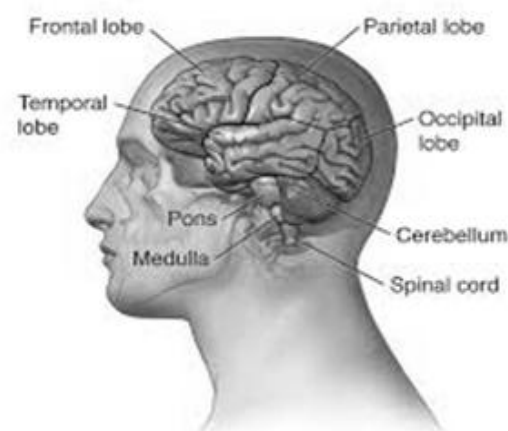

Fig.1 Anatomy of the Brain

\subsubsection{Cerebrum}

The cerebrum is a large part of a brain and it contains cerebral cortex and several sub-cortical structures like hippocampus, basal ganglia and olfactory bulb. It controls all voluntary actions in the 
body. The cerebral cortex is the 3 millimeters outer layer of gray matter over the hemispheres and it is divided into right and left cerebral hemispheres, which controls most of our body functions, including the mysterious state of consciousness, the senses, the body's motor skills, reasoning and language. Sphere is divided into frontal, temporal, parietal, each hemi and occipital lobes. In ADHD affects brain, cerebral cortex does not function and it leads to slow motor activity.

\subsubsection{Frontal Lobe}

The frontal lobe plays an important role to decision Making, pay attention to a task and to complete. Further, normally frontal lobes play a proper role in the situation behavior and emotional impulse control. In ADHD affected brains, the frontal lobe does not function properly and leads to learning disability.

\subsubsection{Hippocampus}

The hippocampus is a brain part which responsible for processing of long term memory and emotional reaction. In ADHD children, hippocampus may lead to memory loss and disorientation which are the basic symptoms of any disease.

\subsubsection{Thalamus}

The thalamus acts as a relay station and it will receive sensory signals from the sensory systems, process and send to cerebral cortex where they are integrated with the conscious mind and it controls conscious state. In ADHD children, Thalamus may direct to poor conscious.

\subsubsection{Cortexes}

The inhibitory mechanisms of the cortex are sort of like the body's impulse control center. A proper functioning of cortex results in a "reining in" of hyperactivity and/or angry outbursts, for example. In an ADHD brain, the inhibitory mechanisms of the cortex do not function properly, resulting in little or no impulse control in certain situations.

\subsubsection{Limbic System}

Limbic system will be more responsible to alert or alarm the people from dangerous situations. Persons with faulty limbic systems may be subject to emotional outbursts or be oversensitive to their environment. ADHD may be affects energy levels as well as sleep patterns and stress supervision.

\subsubsection{Reticular Activating System (RAS)}

RAS is the attention center in the brain and it seems to be the center of motivation. It controls walking and sleeping patterns, have an ability to concentrate and focus attention. ADHD appears that the reticular activating system cannot keep up with the demands placed on it. This leads to over-arousal, under-motivation. Finally children with ADHD, their brain parts are function not properly and it is unconditional stage. To identify abnormal parts, Medical image segmentation is used.

\subsection{MEDICAL IMAGE SEGMENTATION}

The brain images are acquired from various imaging techniques such as Positron Emission Tomography (PET), Magnetic Resonance Imaging (MRI), and Computed Tomography (CT). Analysis of abnormal brain parts is the main task for diagnosing the diseases like brain tumor, stroke, paralysis, Epilepsy, ADHD, and Alzheimer's disease etc. To diagnose ADHD, MRI brain images are used in this research work.

\subsection{Magnetic ReSONANCE IMAGING}

Magnetic Resonance Imaging is a highquality medical imaging technique used to produce the detailed images of the brain and the brain stem. It provides information about the anatomical structure with high contrast of soft tissues and spatial resolution which is used to find problems such as tumors, bleeding, brain injury, blood vessel diseases, or infection. MRI images provide an efficient way to segregate white matter, grey matter and Caudate Nucleus and Cerebrospinal Fluid which cause ADHD more clearly. The different views of the brain internal structure, namely sagittal (lateral plane), coronal (frontal/transverse), and horizontal (axial view) are shown in Fig 2 and Fig 3. The axial view contains frontal horns, Caudate nucleus, Putamen, Globus Pallidus and etc. The caudate Nucleus is extracted from the axial view of MRI brain image and depends upon the caudate nucleus ADHD is diagnosed [2].

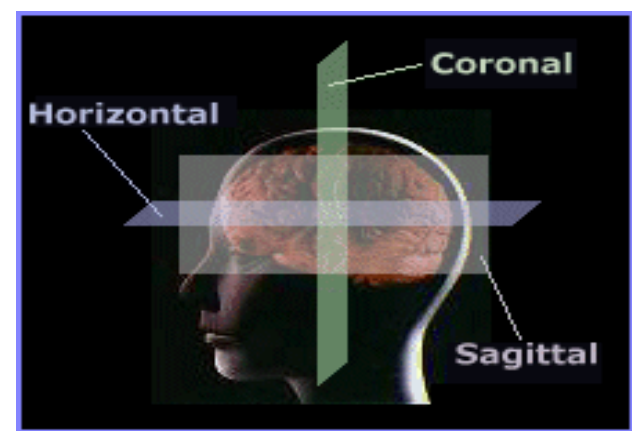

Fig.2 MRI Slice Orientations
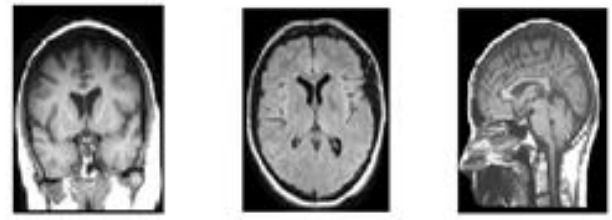

(a)

(b)

(c) 
Fig.3 (a) Coronal (b) Axial (c) Sagittal

This paper is organized into $\mathrm{V}$ sections. The review of literature survey is provided in Section II. Segmentation Techniques to extract caudate Nucleus is proposed in Section III. Experimental results and analysis are presented in Section IV. Conclusion is made in Section V.

\section{LITERATURE SURVEY}

A.S.Bhide, et.al, has proposed Fuzzy C Means Clustering technique to extract brain tumor in MRI brain image. It gives better results with high computational and less memory requirement[3]. Rohini Paul Joseph et.al, proposed K - means clustering algorithm followed by morphological filtering to extract the brain tumor. It gives accurate results since morphological filtering has reduced the mis-clustered regions effectively. Usage of K-means algorithm leads to less computing time [8].

E.A.Zanaty et.al enforced a hybrid approach which combines three algorithms fuzzy clustering, seed region growing, and Jaccard similarity coefficient algorithm to determine gray (GM) and white matter tissue (WM) volumes. It produces accurate and stable results[6]. G. Evelin Sujji proposed MRI Brain Image Segmentation method based on Threshold to segment the regions White Matter, Grey Matter, and Cerebrospinal Fluid[9].

Laura Igual et.al, proposed an automatic method for external and internal segmentation of caudate nucleus based on Machine Learning methodologies. A novel reliable Caudate Cut method combined with atlas-based segmentation strategy with the Graph Cut energy-minimization framework was proposed to segment the small and low-contrast caudate structure by Igual L[10]. Ramgopal Kashyap et.al, proposed "Modified Region Based Segmentation to detect and segment parts of interest in medical images. It gives better performance as well as accuracy [11].

In this paper, three segmentation techniques namely Fuzzy C-Means, Region Based Active Contour and Threshold based Segmentation are proposed to extract the Caudate Nucleus.

\section{PROPOSED METHODOLOGY}

The initial task of the diagnosis of ADHD is to identify the brain abnormality i.e., to identify the abnormal regions of the brain. The proposed system has five modules: Image Acquisition, Preprocessing, Segmentation and the flow diagram is shown in Fig 3 .

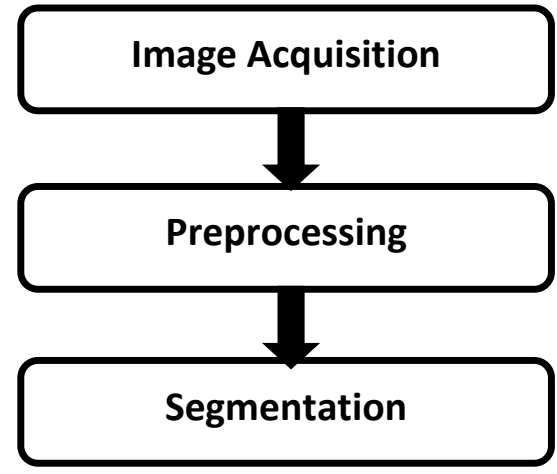

Fig.3 Flow Diagram of the Proposed Methodology

\subsection{Image Acquisition}

The brain MRI images are collected from various mind care hospitals. These images are not directly suitable for the diagnosis of ADHD, because the interior portion of this image is not clear and clarity. So, we have to put the MRI scan image on fluorescent viewing box to improve the clarity of the interior portion of the MRI image and converted to a specific format including BMT, JPEG.

\subsection{Pre-processing}

The captured MRI image may be corrupted with some undesired noises. To remove noises and to enhance the images Median filtering is applied in the preprocessing phase. Median Filtering is a nonlinear method to remove noise from images and it works by moving though the image pixel by pixel, replacing each value with the median value of neighboring pixels. The preprocessed image is shown in Fig 4. The goal of Median Filtering Techniques will increase the accuracy and clarity of the brain images. Finally, the original image is resized into $256 \times 256$.

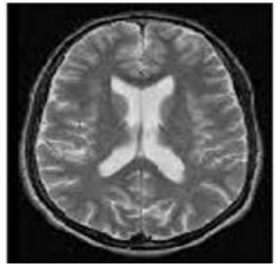

a) Original Image

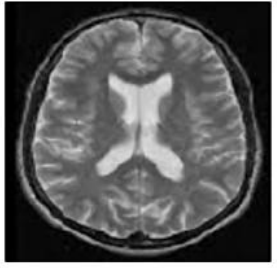

b) Preprocessed image Fig.4 Image Preprocessing

\subsection{Segmentation Techniques}

In this work, three segmentation techniques namely Fuzzy C-Means Segmentation, Region Based Active contour Segmentation, Threshold Based Segmentation are proposed to extract caudate nucleus from brain MRI images. 


\subsubsection{Fuzzy C-Means Segmentation (FCM)}

Fuzzy c-means (FCM) clustering algorithm is an unsupervised clustering technique in the field of medical imaging. In FCM, each pixel is assigned a membership value which ranges from 0 to 1 . In general, the member of one fuzzy set can also be a member of another fuzzy set in the same image because Fuzzy clustering by contrast will allow data points to belong to more than one group. The data is partitioned into two groups such that the degree of association is strong within one group and weak between data in different groups. Fuzzy partition is carried out by an iterative optimization of object with cluster center. Compare to K-means, FCM provides better results for overlapped regions and data points which belong to more than one cluster. So that FCM is popularly used for soft segmentations like brain tissue model. The procedure for the proposed Fuzzy C-mean algorithm is represented as below.

Step 1: Select the image and get the size of the image.

Step 2: Define the number of iterations and number of clusters.

Let $X=\left\{x_{1}, x_{2}, x_{3} \ldots, x_{n}\right\}$ be the set of data points and $V=\left\{v_{1}, v_{2}, v_{3} \ldots, v_{c}\right\}$ be the set of centers.

Step 3: Convert the input image into vectors.

Step 4: Randomly select the center points 'c' for each cluster.

Step 5: Calculate the fuzzy membership value ' $\mu \mathrm{ij}$ ' using equation (1)

ij $=1 / \sum_{k=1}^{0}\left(d_{i j} / d_{i k}\right)^{(2 / m-1)}$

Step 6: Compute the fuzzy centers 'vj' using equation (2)

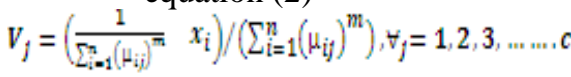

Step 7: Repeat steps 5 and 6 until a minimum value ' $\mathrm{j}$ ' is achieved.

Main objective of fuzzy c-means algorithm is to minimize:

$$
I\left(U_{v} V\right)=\sum_{i=1}^{n} \sum_{j=1}^{e}\left(\mu_{i j}\right)^{m}\left\|x_{i}-v_{j}\right\|^{2}
$$

Where,

$$
\begin{aligned}
& \mathrm{n} \text { - Number of data points. } \\
& \mathrm{m} \text { - Constant value } \\
& \mathrm{c} \text {-Number of clusters } \\
& \mu_{\mathrm{ij}} \text {-Membership value of } \mathrm{x}_{\mathrm{i}} \text { with } \mathrm{i}^{\text {th }} \text { cluster } \\
& \text { center. } \\
& \mathrm{v}_{\mathrm{j}-}-\mathrm{j}^{\text {th }} \text { cluster center } \\
& \mathrm{d}_{\mathrm{ij}}-\text { Euclidean distance between } \mathrm{i}^{\text {th }} \text { data and } \mathrm{j}^{\text {th }} \\
& \text { cluster center. } \\
& \left\|\boldsymbol{x}_{\tilde{i}}-\boldsymbol{v}_{\tilde{j}}\right\| \text {-Distance between one of the } \mathrm{n}
\end{aligned}
$$

data points $\mathrm{x}_{\mathrm{i}}$ and closest cluster center $\mathrm{v}_{\mathrm{j}}$.

The results of Fuzzy C-Means algorithm is shown in Fig 4.

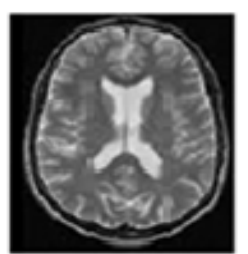

a) Original Image b) Extract Caudate Nucleus

Fig.4 Fuzzy C-Means Segmentation

\subsubsection{Region Based Active Contour Segmentation (RBACS)}

Region based segmentation is a method to partition an image into regions. Active contour with Chan-Vese model is proposed to extract the caudate nucleus. The model is based on an energy minimization problem, which can be reformulated in the level set formulation, leading to an easier way to solve the problem. It evolves the segmentation using an iterative process. By default, it performs 100 iterations. Mask is a binary image that specifies the initial state of the active contour. The boundaries of the object regions (white) in mask define the initial contour position used for contour evolution to segment the image. The output image is a binary image where the foreground is white (logical true) and the background is black (logical false). The procedure for the proposed Active contour algorithm is represented as below.

Step 1: Select the MRI Image.

Step 2: Set the mask size which depends on the size of the original image. Mask is created manually or by the built-in function.

Step 3: Get the distance map of the initial mask.

Step 4: Set the value of level set function.

$$
\begin{aligned}
& \mathrm{C}=\{(\mathrm{x}, \mathrm{y}) \in \mathbb{R}: \Phi(\mathrm{x}, \mathrm{y})=0\} \\
& \operatorname{in}(C)=\{(x, y) \in \mathbb{Q}: \mathbb{x}(x, y)>0\} \\
& \operatorname{out}(C)=\left\{\left(x_{y}, y\right) \in \mathbb{N}, \Phi\left(x_{0}, y\right)<0\right\}
\end{aligned}
$$

From equations (4), (5) and (6), where $\mathrm{C}$ is a spline curve, and $(\mathrm{x}, \mathrm{y})$ is point.

Step 5: Define the number of iterations.

Step 6: Calculate the average intensities $\mathrm{C} 1$ and $\mathrm{C} 2$ by the equations (7) and (8) respectively.

$$
\begin{aligned}
& C_{1}(\Phi)=\frac{\int_{\Omega} u_{0}(x, y) H(\Phi(x, y)) d x d y}{\int_{\Omega} H(\Phi(x, y)) d x d y}
\end{aligned}
$$

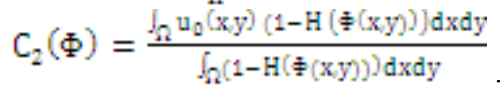

Step 7: Repeat steps 6 and 7 until the object boundary is reached.

The caudate Nucleus extracted by the Region based Active Contour segmentation technique is shown in Fig 5. 


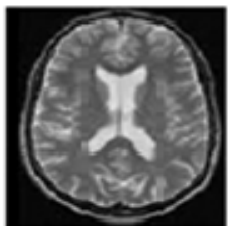

a) Original Image

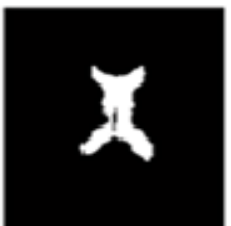

b) Extract Caudate Nucleus Fig.5 Region Based Segmentation

\subsubsection{Threshold Based Segmentation (TBS)}

Threshold is a simple segmentation technique used to extract the object from the image. The caudate nucleus can be extracted from the brain image using certain threshold value

Threshold operation is a grey value remapping operation denoted by

$$
\mathrm{F}(G)= \begin{cases}0 & \text { if } \mathrm{f}(\mathrm{x}, \mathrm{y})<\mathrm{T} \\ 1 & \text { if } \mathrm{f}(\mathrm{x}, \mathrm{y}) \geq \mathrm{T}\end{cases}
$$

Where, $f(x, y)$ represents a grey value and $T$ is the threshold value. After Thresholding, the image has been segmented into two parts using equation (9), identified by pixel value 0 and 1 respectively. The algorithm for Threshold method is represented as below.

Step 1: Read the Brain MRI Image.

Step 2: Assign Initial estimate value to T.

Step 3: Segment the region of interest.

Threshold based segmentation result is shown in Fig 6.

After Segmentation, the image may consist of unwanted pixels i.e., the regions other than caudate nucleus. I.e., if the threshold value is not selected correctly, the results may give under segmentation. So, Morphological Filtering is applied to remove the unwanted pixels.
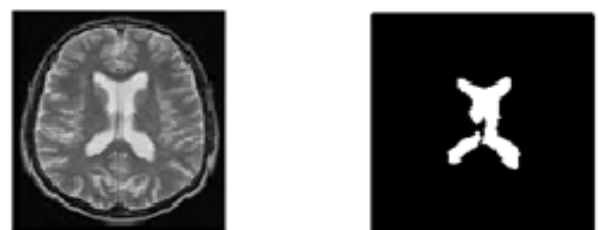

a) Original Image b) Extract Caudate Nucleus

Fig.6 Threshold Based Segmentation

\subsubsection{Morphological Filtering}

Morphological Filters are formed from the basic morphology operations such us erosion, dilation, opening and closing. It is a collection of non-linear operations related to the shape or features in an image. In this paper, opening operation is used to extract the caudate nucleus of MRI brain image without unwanted pixels.

The opening of an image $f$ by a structuring element $s$ is denoted by erosion followed by dilation: $(\mathrm{f} \Theta \mathrm{s}) \oplus \mathrm{s}=> \begin{cases}0 & \text { if foreground } \leq \mathrm{s} \\ 1 & \text { if foreground }>\mathrm{s}\end{cases}$

Finally in the target image, Foreground structures that are smaller than the structure element will be strayed and larger structures will be retained by equation (10).

From this result, Fuzzy C-Means Segmentation gives perfect results compared to other segmentations (Region Based Active Contour and Threshold based segmentation). FCM clustering model directly extract the caudate nucleus from MRI brain images. But, Region Based Active contour Model and Threshold based segmentation model requires post processing as morphological filtering to retain only the Caudate Nucleus.

\section{EXPERIMENTAL ANALYSIS \& RESULTS}

The proposed method was tested on 20 real images, out of which 7 images are taken for discussion. The input MRI images and the results of three segmentation techniques are listed in the Table I. Region based and Threshold segmentation techniques have a problem of over and under segmentation compared to FCM Clustering Technique. The proposed segmentation models are compared based on the attributes Region continuity, speed, Noise immunity and accuracy and given in Table II.

TABLE I: RESULTS OF PROPOSED IMAGE SEGMENTATION TECHNIQUES

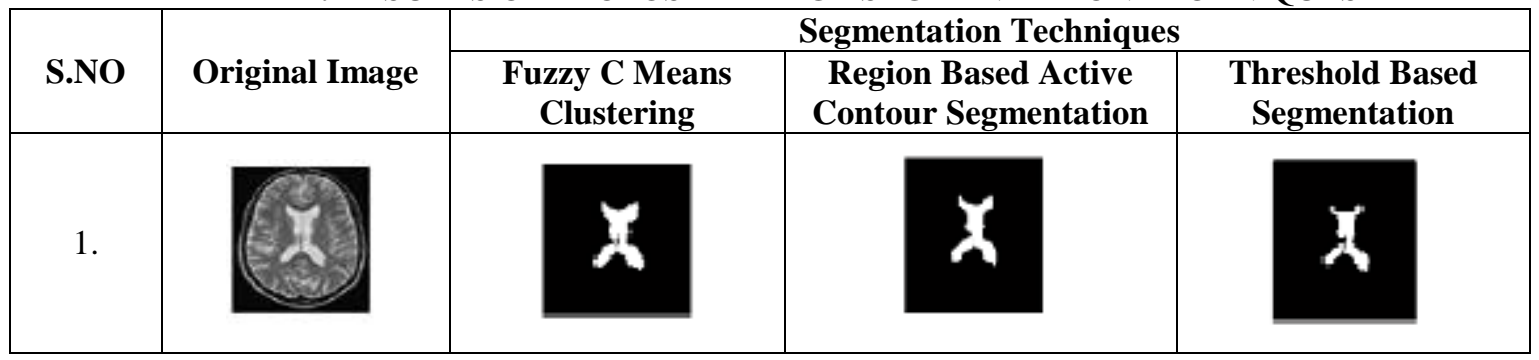




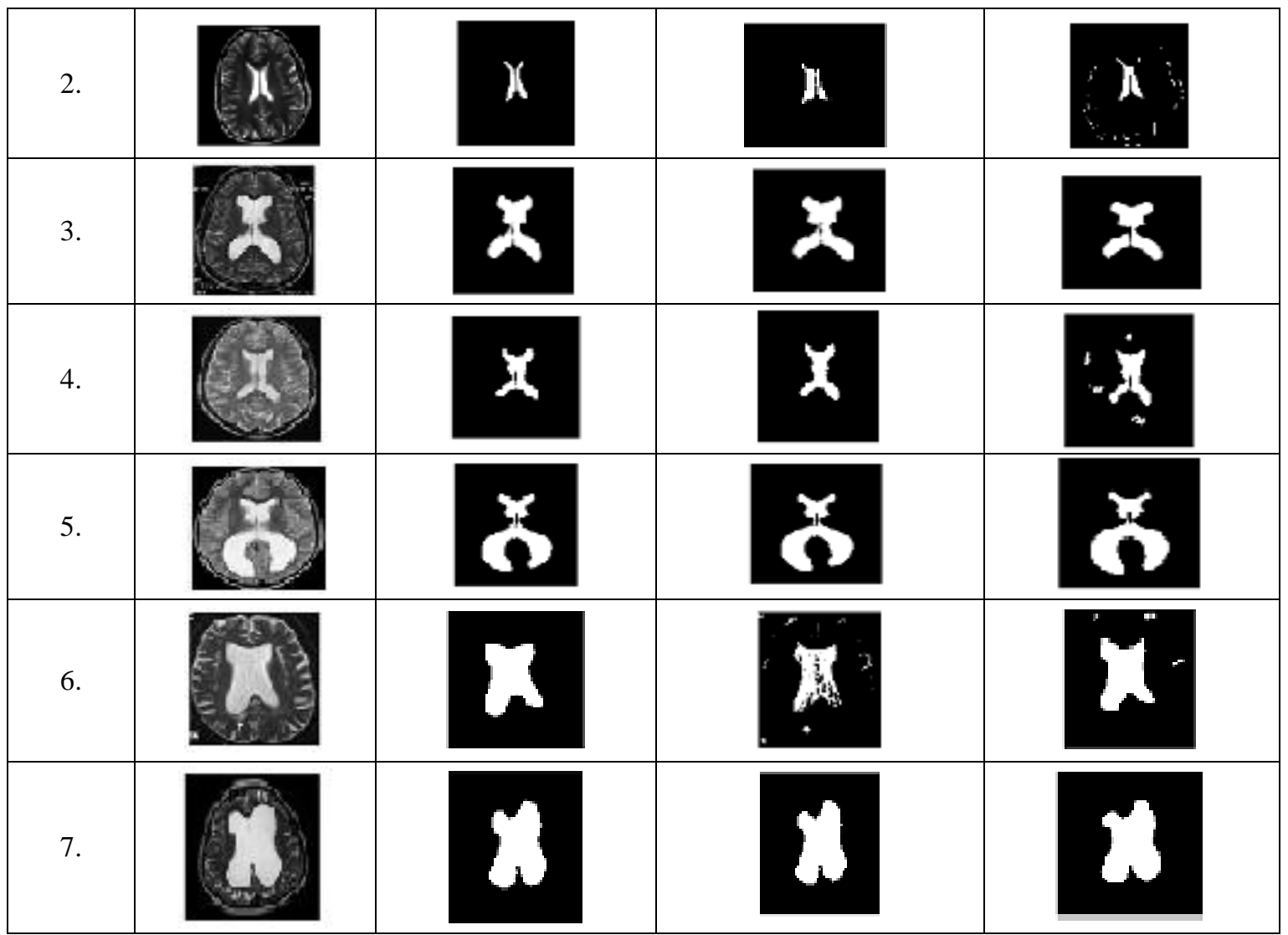

TABLE II: COMPARISON OF SEGMENTATION MODELS

\begin{tabular}{|c|c|c|c|c|}
\hline $\begin{array}{c}\text { SEGME } \\
\text { NTATI } \\
\text { ON } \\
\text { MOD } \\
\text { ELS }\end{array}$ & $\begin{array}{c}\text { REGION } \\
\text { CONTIN } \\
\text { UITY }\end{array}$ & SPEED & $\begin{array}{c}\text { NOISE } \\
\text { IMMU } \\
\text { NITY }\end{array}$ & $\begin{array}{l}\text { ACCU } \\
\text { RACY }\end{array}$ \\
\hline FCM & High & $\begin{array}{c}\text { Fast } \\
(2.8713 \\
\text { Sec })\end{array}$ & Less & $\begin{array}{c}\text { Very } \\
\text { Good } \\
(100 \%)\end{array}$ \\
\hline $\begin{array}{c}\text { RBAC } \\
\mathrm{S}\end{array}$ & High & $\begin{array}{c}\text { Slow } \\
(41.376 \\
\text { Sec })\end{array}$ & $\begin{array}{c}\text { Mediu } \\
\mathrm{m}\end{array}$ & $\begin{array}{l}\text { Good } \\
(71 \%)\end{array}$ \\
\hline TBS & $\begin{array}{c}\text { Moderat } \\
\mathrm{e}\end{array}$ & $\begin{array}{c}\text { Fast } \\
(1.9482 \\
\text { Sec })\end{array}$ & Less & $\begin{array}{c}\text { Moderat } \\
\mathrm{e} \\
(57 \%)\end{array}$ \\
\hline
\end{tabular}

From the Table II, it has been identified that the FCM clustering model effectively extracts the caudate nucleus from the brain images. And at the same time Threshold based segmentation model effectively segments the regions but very poor to extract the abnormal features from the images.

\section{CONCLUSION}

One of the main causes for the occurrence of ADHD is the brain damage or brain injury. Any brain damage or brain injury may cause abnormality in the growth of caudate nucleus. So to predict the prevalence of ADHD, three different segmentation techniques namely Fuzzy C-Means Segmentation, Region Based Segmentation and Threshold Based Segmentation are proposed in this research work to

extract the caudate nucleus from the MRI brain images. The segmentation results are compared and analyzed based on various performance metrics and found that the Fuzzy C-Means Segmentation algorithm performs well in terms of region continuity, computation time, and accuracy.

\section{REFERENCES}

[1] Larry J. Seidman, Eve M. Valera, and Nikos Makris "Structural Brain Imaging of Attention-Deficit/Hyperactivity Disorder" BIOL PSYCHIATRY (2005)57:1263-1272.

[2] Amy L. Krain a, F. Xavier Castellanos "Brain development and ADHD" Clinical Psychology Review 26 (2006),433444,doi:10.1016.

[3] Shally HR, Chitharanjan K, "Tumor Volume Calculation Of Brain From MRI Slices", International Journal of Computer Science \& Engineering Technology (IJCSET), ISSN: 2229-3345 Vol. 4 No. 08 Aug 2013.

[4] Prof. A.S.Bhide, PriyankaPatil, ShraddhaDhande, "Brain Segmentation using Fuzzy $\mathrm{C}$ means clustering to detect 
tumor Region" International Journal of Advanced Research in Computer Science and Electronics Engineering, Volume 1, ISSN: 2277 - 9043 , Issue 2, April 2012.

[5] Rohini Paul Joseph, C. Senthil Singh, M.Manikandan3 "Brain Tumor MRI Image Segmentation and Detection in Image Processing" IJRET: International Journal of Research in Engineering and Technology eISSN: 2319-1163 | pISSN: 2321-7308.

[6] Zanaty, "Determination of Gray Matter (GM) and White Matter (WM) Volume in Brain Magnetic Resonance Images (MRI)" International Journal of Computer Applications (0975 - 8887) Volume 45No.3, May 2012.

[7] SivaSankari.S, Sindhu.M, Sangeetha.R , ShenbagaRajan A "Feature Extraction of Brain Tumor Using MRI" International Journal of Innovative Research in Science, Engineering and Technology (An ISO 3297: 2007 Certified Organization) Vol. 3, Issue 3, March 2014

[8] G. Evelin Sujji1, Y.V.S. Lakshmi2 , G. Wiselin Jiji3, "MRI Brain Image Segmentation based on Thresholding" International Journal of Advanced Computer Research (ISSN (print): 2249-7277 ISSN (online): 2277-7970) Volume-3 Number-1 Issue-8 March-2013.

[9] Laura Iguala,b, Joan CarlesSolivac, Sergio Escaleraa, Roger Gimenoa,3, Oscar
Vilarroyac,1, Petia Radevaa,b,4 "Automatic brain caudate nuclei segmentation and classification in diagnostic of AttentionDeficit/Hyperactivity Disorder” 0895-6111, 2012 Elsevier Ltd.

[10] Usha Mittal,Sanyam and "Effect of Morphological Filters on Medical Image Segmentation using Improved Watershed Segmentation" International Journal of Computer Science \& Engineering Technology (IJCSET), ISSN : 2229-3345 ,Vol. 4 No. 06 Jun 2013.

[11] Laura Iguala, Joan Carles Solivac, 1, Sergio Escaleraa,b,2, Roger Gimenoa, Oscar Vilarroyac, PetiaRadevaa "Automatic brain caudate nuclei segmentation and classification in diagnostic of AttentionDeficit/Hyperactivity Disorder" Elsevier Ltd (2012) 591-600.

\footnotetext{
International Journal of Engineering Research and Applications (IJERA) is UGC approved Journal with S1. No. 4525, Journal no. 47088. Indexed in Cross Ref, Index Copernicus (ICV 80.82), NASA, Ads, Researcher Id Thomson Reuters, DOAJ.

I.Jemima Bibiyana. "Diagnosis and Inference of ADHD ." International Journal of Engineering

Research and Applications (IJERA) 7.7 (2017): 77-83.
} 